\title{
Convergent flow in a two-layer system and plateau development
}

\author{
Julio Gratton ${ }^{1, a)}$ and Carlos Alberto Perazzo ${ }^{2, b)}$ \\ ${ }^{1}$ Departamento de Física, Facultad de Ciencias Exactas y Naturales, INFIP-CONICET, Universidad de \\ Buenos Aires, Ciudad Universitaria, Pab. I, 1428 Buenos Aires, Argentina \\ ${ }^{2}$ Departamento de Física y Química, Universidad Favaloro, Solís 453, 1078 Buenos Aires, Argentina
}

(Received 6 December 2010; accepted 25 March 2011; published online 11 April 2011)

\begin{abstract}
In order to describe the development of plateaus such as the Tibet and the Altiplano we extend the two-layer model used in a previous paper [C. A. Perazzo and J. Gratton, Phys. Fluids 22, 056603 (2010)] to reproduce the evolution of mountain ranges. As before, we consider the convergent motion of a system of two liquid layers to simulate the crust and the upper mantle that form a lithospheric plate, but now we assume that the viscosity of the crust falls off abruptly at a specified depth. We derive a nonlinear differential equation for the evolution of the thickness of the crust. The solution of this equation shows that the process consists of a first stage in which a peaked range is formed and grows until its root reaches the depth where its viscosity drops. After that the range ceases to grow in height and a flat plateau appears at its top. In this second stage the plateau width increases linearly with time as its sides move outward as traveling waves. We derive simple approximate formulas for various properties of the plateau and its evolution. (C) 2011 American Institute of Physics. [doi:10.1063/1.3578481]
\end{abstract}

\section{INTRODUCTION}

Mountains and high plateaus are striking features of our planet and their origin and evolution have been investigated for a long time. It is known that the lithosphere (the outer solid layer of the Earth) is a two-layer structure in which the crust rests on the denser upper mantle, being separated by the Mohorovičić discontinuity (called Moho). The lithosphere is divided into several approximately rigid plates that rest on the hotter and more fluid asthenosphere. The relative motion of these plates is the cause of orogeny due to the shortening and consequent thickening of the crust that occurs when two continental plates collide or when an oceanic plate is subducted beneath a continent. On the timescale of these processes the lithosphere is in local hydrostatic equilibrium, a condition called isostasy, which implies that the visible regional topography is accompanied by a corresponding antitopography of the Moho, called root.

Clearly orogeny involves many disciplines and interests a broad range of scientists. To attempt a realistic and detailed theoretical description of these processes is an exceedingly complex task because across the lithosphere there are large variations of the temperature, density, and rheological parameters as well as other properties, many of which, to compound the issue, are poorly known. To this we must add the complications due to the geometry and the time dependence of the motion of the plates.

In a recent paper ${ }^{1}$ we investigated the evolution of the topographic relief produced by the convergent motion of a system consisting of two superimposed layers of liquids that differ in density and viscosity to simulate the crust and the upper mantle of two lithospheric plates that are pushed together by basal traction, which we imposed as a boundary

\footnotetext{
${ }^{a)}$ Electronic mail: jgratton@tinfip.lfp.uba.ar. Researcher of CONICET.

${ }^{b)}$ Electronic mail: perazzo@favaloro.edu.ar. Researcher of CONICET.
}

condition at the lithosphere-asthenosphere boundary (LAB). Assuming isostasy, we derived a nonlinear differential equation for the evolution of the thickness of the crust and solved this equation numerically to obtain the profile of the range. We found that a peaked topography is produced. We also found an approximate self-similar solution that describes reasonably well the process and that predicts simple scaling laws for the height and width of the range as well as an expression of the shape of its transversal profile. These theoretical results given by our model were compared with approximately straight segments of several mountains belts and found a good agreement. Notice that in our model we describe the lithosphere as two stacked layers of liquids despite that the most elevated part of the topography may have a brittle elastic and not by ductile strength.

The simple two-layer model in Ref. 1 does not describe, however, the development of structures as the Tibet or the Altiplano, which are almost flat plateaus limited laterally by steep escarpments, as shown in Fig. 1. See also the profiles of the Tibet and the Altiplano shown in Refs. 2 and 3, respectively.

Although there are several proposed mechanisms as responsible of the plateau formation, none of them is generally accepted. However, some researchers ${ }^{4}$ agree with the idea that a plateau is produced when the motion of the upper crust decouples from the basal traction, as happens if a very low viscosity layer lies above the place where the traction is acting. ${ }^{5-10}$ The formation of plateaus due to the presence of a low viscosity layer in the lithosphere has been addressed by several authors by means of the so-called "channel flowextrusion" hypothesis in connection with the Tibet ${ }^{11-16}$ and the Altiplano. ${ }^{17-19}$ In these works it is assumed that the low viscosity layer lies within the lower crust and that the basal traction is exerted at the Moho by the mantle lithosphere, which is otherwise ignored and plays no role in the model. 
(a)

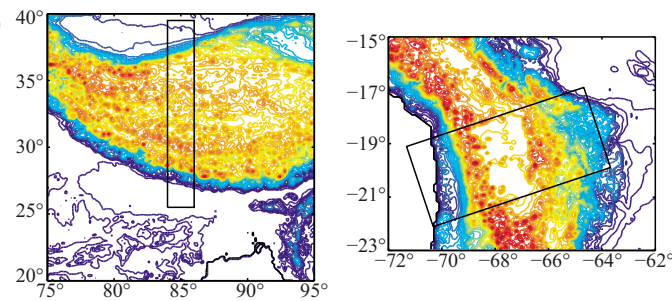

(b)

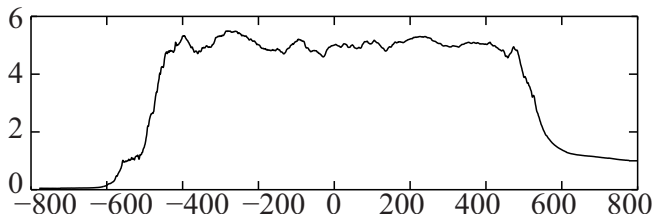

(c)

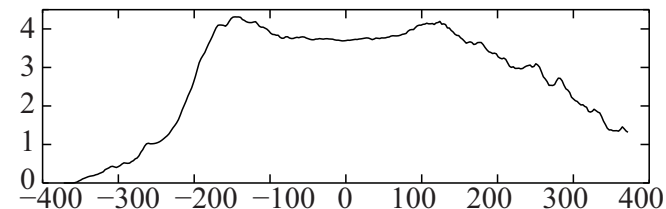

FIG. 1. (Color online) Topography of real plateaus. (a) Contour maps of Tibet (left) and Altiplano (right) from the digital elevation data GTOPO30 (these data are available in the website of U.S. Geological Survey's Earth Resources Observation and Science Center); the rectangles denote the regions where the averages are calculated. (b) and (c) Average profile of Tibet and Altiplano, respectively (the altitude and the horizontal coordinates are in kilometers)

For example, Royden ${ }^{7}$ assumed that the crust is divided into an upper part, whose viscosity is uniform, and a lower part, in which the viscosity falls off exponentially with depth. Then, as the crust thickens the root of the range eventually attains the depth where the viscosity falls off, so that the basal traction is decoupled from the motion of the upper part of the crust. As a consequence the relief ceases to grow in height and a plateau develops and widens as more crustal mass is added by the convergent motion of the plates.

According to the model in Ref. 1 a similar decoupling that may lead to the formation of a plateau occurs only when the root of a range touches the asthenosphere, but a simple calculation shows that this would produce an absurdly high plateau (typical values yield $20 \mathrm{~km}$ ).

In this paper we change slightly the two-layer model in Ref. 1 to include the possibility that the viscosity of the crust falls off abruptly at a specified depth, as it was assumed in the previously mentioned papers. However, it is important to remark that our two-layer model $^{1}$ and its extension in the present paper take into account the dynamics of the upper mantle and not only that of the crust as it is done by other authors that assume that the basal traction acts on the Moho. In consequence, while our results are qualitatively similar to those obtained by other authors, they differ quantitatively as they depend on the lithospheric mantle parameters. Here we keep the model as simple as possible as our aim is to describe the essentials of the process in order to clarify the basic physics involved.

This paper is organized as follows. In Sec. II we present the two-layer model with the changes needed to describe the formation of plateaus. In Sec. III we describe the numerical solutions and show that the development of a plateau is a
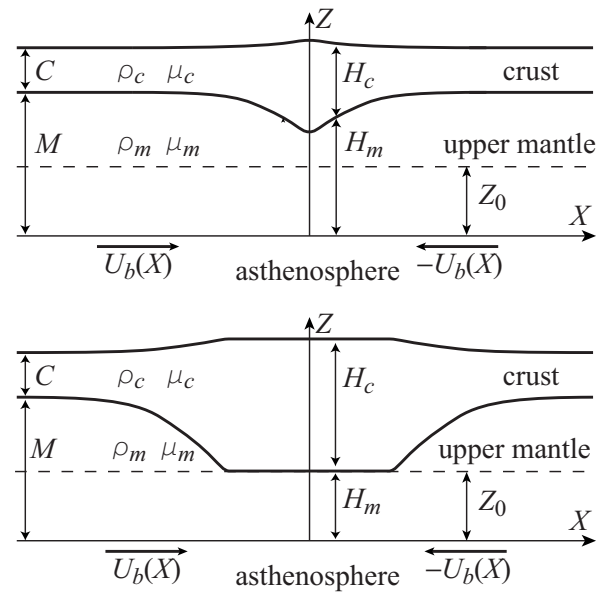

FIG. 2. Geometry of the two-layer model employed to describe the formation of a plateau. The upper figure shows the ridge that appears in the first stage and the lower one shows the plateau that develops in the second stage (see Sec. III) when the root arrives at the low viscosity region of the crust (dashed line).

process consisting of two stages. In the first stage a peaked ridge grows until the root reaches the low viscosity region of the crust. At this moment the second stage begins in which the plateau develops. In Sec. IV we show that during the second stage both sides of the plateau behave as traveling waves. Finally, Sec. V contains the discussion and conclusions.

\section{THE TWO-LAYER MODEL}

In this section we parallel the derivation of the evolution equations obtained in our previous paper, ${ }^{1}$ highlighting the changes introduced to describe the formation of a plateau. We consider a two-layer liquid film as shown in Fig. 2 and we assume for simplicity plane symmetry. The upper layer (the crust) has a density $\rho_{c}$, a thickness $H_{c}(X, T)$, and a viscosity $\mu_{c}$ that is not constant as assumed in Ref. 1 but depends on the vertical coordinate $Z$. The lower layer (the lithospheric mantle) has a density $\rho_{m}$, a thickness $H_{m}(X, T)$, and a viscosity $\mu_{m}$. Typically, for a continental plate $\rho_{c}$ $\approx 2.7 \mathrm{~g} / \mathrm{cm}^{3}$ and $\rho_{m} \approx 3.2 \mathrm{~g} / \mathrm{cm}^{3}$.

Initially, both layers are uniform, with $H_{c}(X, 0)=C$ and $H_{m}(X, 0)=M$. To model the basal traction that is believed to drive the plate motion we assume that at $T=0$ the bottom of the lithosphere $(Z=0)$ starts moving with a prescribed velocity $U_{b}(X)$. We next assume isostasy, which means that for $0 \leq Z \leq H_{m}$ the pressure does not depend on $X$. Notice that this implies that as the thickness of the crust increases, part of the mass of the lithospheric mantle crosses the LAB and passes into the asthenosphere, so that the mass of the lithospheric mantle is not conserved. We assume a slow viscositydominated flow and employ the lubrication approximation (see Refs. 20-22). We neglect inertia and assume that the slope of the free surface is gentle, so that the horizontal components of the velocities of the fluids are much larger than the vertical ones and that their vertical gradients are much larger than the horizontal gradients. In this way the Stokes equation takes the form 


$$
\frac{\partial P}{\partial X}=\mu_{m} \frac{\partial^{2} U}{\partial Z^{2}}, \quad \frac{\partial P}{\partial Z}=-\rho_{m} g
$$

for $0 \leq Z \leq H_{m}$ and

$$
\frac{\partial P}{\partial X}=\mu_{c}(Z) \frac{\partial^{2} U}{\partial Z^{2}}, \quad \frac{\partial P}{\partial Z}=-\rho_{c} g
$$

for $H_{m} \leq Z \leq H_{m}+H_{c}$. In these equations $P$ is the pressure, $U(X, Z, T)$ is the horizontal velocity, and $g$ is the gravity. ${ }^{23}$ The second equations in Eqs. (1) and (2) mean that the pressure is hydrostatic; integrating them and using the isostasy condition $\left(\partial P / \partial X=0\right.$ for $\left.0 \leq Z \leq H_{m}\right)$ we find $\rho_{m} \partial H_{m} / \partial X=$ $-\rho_{c} \partial H_{c} / \partial X$. Integrating this equation and using the initial condition we obtain

$$
H_{m}=M+\frac{\rho_{c}}{\rho_{m}}\left(C-H_{c}\right) .
$$

Using this result to eliminate $H_{m}$ we find a single equation for the dependent variable $H_{c}$.

The first equation in Eq. (1) and the isostasy condition imply that the velocity profile in the lithospheric mantle is linear. To complete the derivation of the velocity profile we assume that $U(Z=0)=U_{b}(X)$ is the prescribed basal velocity, which the velocity and the shear stress are continuous at $Z$ $=H_{m}$ and which the shear stress vanishes at $Z=H_{m}+H_{c}$. Then we integrate twice the first equations in Eqs. (1) and (2) with respect to $Z$ to obtain

$$
U= \begin{cases}U_{b}-\frac{g \rho_{c}}{\mu_{m}} H_{c}\left(\frac{\partial H_{c}}{\partial X}+\frac{\partial H_{m}}{\partial X}\right) Z, & 0 \leq Z \leq H_{m} \\ U_{b}+g \rho_{c}\left(\frac{\partial H_{c}}{\partial X}+\frac{\partial H_{m}}{\partial X}\right)\left[-\left(H_{c}+H_{m}\right) \int_{H_{m}}^{Z} \frac{d Z^{\prime}}{\mu_{c}\left(Z^{\prime}\right)}+\int_{H_{m}}^{Z} \frac{Z^{\prime} d Z^{\prime}}{\mu_{c}\left(Z^{\prime}\right)}-\frac{H_{c} H_{m}}{\mu_{m}}\right], & H_{m} \leq Z \leq H_{m}+H_{c} .\end{cases}
$$

Notice that, in general, the velocity profile in the crust is not parabolic except when $\mu_{c}$ is a constant as in Ref. 1.

We then define the vertically averaged horizontal velocity in the crust as

$$
V_{c}=\frac{1}{H_{c}} \int_{H_{m}}^{H_{m}+H_{c}} U d Z
$$

We set $U_{b}(X)=U_{0} u(X)$, where $U_{0}$ is the maximum basal velocity so that $|u| \leq 1$. Next we introduce the following dimensionless quantities:

$$
\begin{aligned}
& h=H_{c} / C, \quad h_{m}=H_{m} / C, \quad v=V_{c} / U_{0}, \\
& x=X / X_{0}, \quad z=Z / C, \quad t=T U_{0} / X_{0} .
\end{aligned}
$$

We also describe the dependence of $\mu_{c}$ on the dimensionless vertical coordinate $z$ by means of

$$
\mu_{c}=\mu_{0} \phi(z), \quad 0<\phi \leq 1 .
$$

In Eq. (6) the horizontal scale $X_{0}$ is given by

$$
X_{0}=\left(1-\frac{\rho_{c}}{\rho_{m}}\right) \frac{\rho_{c} g M C^{2}}{\mu_{m} U_{0}} .
$$

Finally, inserting the second equation of Eq. (4) in Eq. (5) and using Eq. (6), we obtain

$$
\begin{aligned}
v= & u-\left(1+\frac{\rho_{c} C}{\rho_{m} M}\right) h \frac{\partial h}{\partial x}+\frac{\rho_{c} C}{\rho_{m} M} h^{2} \frac{\partial h}{\partial x}+\frac{\mu_{m}}{\mu_{0}}\left\{\frac{C}{M h} J_{2}\right. \\
& \left.-\left[\frac{1}{h}\left(1+\frac{\rho_{c} C}{\rho_{m} M}\right)+\frac{C}{M}\left(1-\frac{\rho_{c}}{\rho_{m}}\right)\right] J_{1}\right\} \frac{\partial h}{\partial x},
\end{aligned}
$$

where

$$
J_{1}=\int_{h_{m}}^{h+h_{m}} \int_{h_{m}}^{z} \frac{d z^{\prime} d z}{\phi\left(z^{\prime}\right)}, \quad J_{2}=\int_{h_{m}}^{h+h_{m}} \int_{h_{m}}^{z} \frac{z^{\prime} d z^{\prime} d z}{\phi\left(z^{\prime}\right)}
$$

are functions of $h$.

From isostasy condition (3) we have

$$
h_{m}=\frac{M}{C}+\frac{\rho_{c}}{\rho_{m}}(1-h) \text {. }
$$

Then Eq. (9) can be expressed in terms only of $h$. Equations (9)-(11) together with the continuity equation

$$
\frac{\partial h}{\partial t}+\frac{\partial(v h)}{\partial x}=0
$$

govern the dimensionless thickness of the crust when the viscosity of the crust changes with depth, provided $\phi(z)$ is a known function. The visible dimensionless topography $r$ is given by

$$
r \equiv \frac{H_{m}+H_{c}-(M+C)}{C}=\left(1-\frac{\rho_{c}}{\rho_{m}}\right)(h-1) .
$$

To describe the convergence of two plates we assume $u(x)=1$ for $x<0$ and $u(x)=-1$ for $x>0$ as in Ref. 1 . In this way the thickness of the crust starts to increase in the region of convergence. The initial condition is $h(x, 0)=1$, and the boundary conditions are $h( \pm \infty, t)=1$. At $x=0$ we impose the continuity of $h$ and $v$.

In the process we are describing that the mass of the crust is conserved, but as said before this is not true for the lithospheric mantle. It is easy to show that the flow rate $Q$ (per unit of transversal length) of lithospheric mantle material through the $\operatorname{LAB}(z=0)$ is 


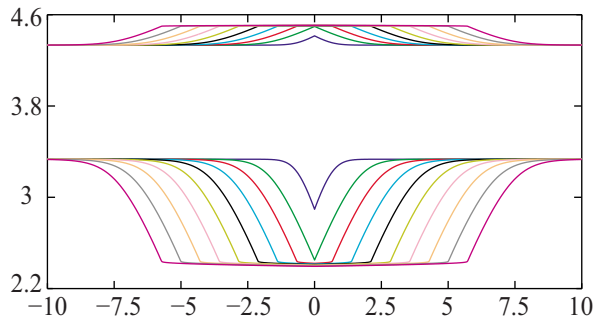

FIG. 3. (Color online) Numerical solutions of Eqs. (9)-(12) and (15) for ten equispaced times between $t=0.24$ and $t=7.44$. It can be appreciated that initially a ridge is formed and grows until the root touches the low viscosity region. After that a plateau develops.

$$
Q=2 U_{0} M\left(1+\frac{\rho_{c} C}{\rho_{m} M}\right) .
$$

Notice that $Q$ is larger than the lithospheric mantle mass inflow $2 U_{0} M$.

To describe the decoupling that leads to the formation of a plateau we assume that the viscosity of the crust falls abruptly around $Z=Z_{0}$ (see the dashed line in Fig. 2) by a few orders of magnitude. To facilitate the numerical calculations it is also convenient to chose $\phi(z)$ in order to obtain closed expressions for $J_{1}$ and $J_{2}$. To take into account these requirements we have assumed in the numerical calculations

$$
\phi(z)=\frac{\phi_{0}(n+1)+\phi_{0}(n-1)\left(z / z_{0}\right)^{n}}{(n+1)+\phi_{0}(n-1)\left(z / z_{0}\right)^{n}} .
$$

In this formula $z_{0}=Z_{0} / C, n$ governs the thickness of the transition and $\phi_{0}=\phi(0)$. We shall assume that $n \gg 1$ and $\phi_{0} \ll 1$ so that the viscosity reduction is abrupt and large. The choice [Eq. (15)] was made for convenience, but any other function with a similar behavior can be used.

\section{PLATEAU FORMATION}

Equations (9)-(15) must be solved numerically. As an example we show in Fig. 3 some solutions for $C=30 \mathrm{~km}$, $M=100 \mathrm{~km}, \quad \rho_{c}=2700 \mathrm{~kg} / \mathrm{m}^{3}, \quad \rho_{m}=3200 \mathrm{~kg} / \mathrm{m}^{3}, \quad$ and $\mu_{0} / \mu_{m}=10$ (the values of these parameters are not essential for the formation of the plateau, in particular, it is not necessary to have $\mu_{0}>\mu_{m}$ ). We have also assumed $n=1000$, $z_{0}=2.4333$, and $\phi_{0}=10^{-5}$ (the effect of different values of $n$ and $\phi_{0}$ will be discussed later).

The most relevant result of the calculations is that a plateau is produced. However, the plateau does not appear from the beginning. Initially a ridge is formed and grows as described approximately ${ }^{1}$ by the self-similar solution $r_{s s}$ given by

$$
r_{s s}(t)=\left(1-\frac{\rho_{c}}{\rho_{m}}\right) \frac{2 \sqrt{t}}{\sqrt{1+\alpha}} f(\psi),
$$

where

$$
f(\psi)=\frac{e^{-\psi^{2}}}{\sqrt{\pi}}-\psi \operatorname{erfc}(\psi), \quad \psi \equiv \frac{x}{2 \sqrt{(1+\alpha) t}},
$$

erfc is the complementary error function, and $\alpha$ $=\mu_{m} C / 3 \mu_{0} M$.

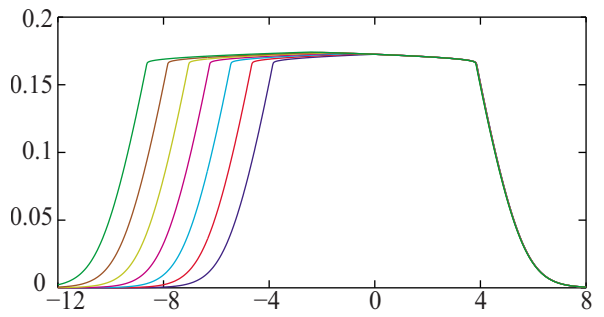

FIG. 4. (Color online) The sides of a plateau move as traveling waves. Plots of the topography $r$ vs $x-u_{f}\left(t-t_{i}\right)$ with $u_{f}$ given by Eq. (19) and $t_{i}=5.36$ for $t=5.36,5.8,6.24,6.68,7.12,7.56$, and 8 . As all the right sides overlap on a unique curve we infer that the right side moves with the constant speed $u_{f}$ without changing its shape.

When the bottom of the root of the ridge arrives at $z_{0}$ this first stage ends, the ridge ceases to grow, and a second stage begins in which a flat top appears and widens, thus producing a plateau as all the added mass accumulates at the sides of the relief. We call $t_{0}$ the moment when the plateau begins to develop.

The height $r_{0}$ of the plateau is determined by $z_{0}$ according to

$$
r_{0}=\left(\frac{\rho_{m}}{\rho_{c}}-1\right)\left(\frac{M}{C}-z_{0}\right) .
$$

\section{PLATEAU EVOLUTION AS A TRAVELING WAVE}

The profiles in Fig. 3 strongly suggest that during the evolution of the plateau (second stage, $t>t_{0}$ ) each slope moves with a constant speed, which we shall call $u_{f}$ (thus the rate of widening is $2 u_{f}$ ), and maintains the shape it had at $t_{0}$. If this was true the conservation of crustal mass yields

$$
u_{f}=\frac{\rho_{c}}{\rho_{m}\left(M / C-z_{0}\right)}=\frac{\rho_{m}-\rho_{c}}{\rho_{m} r_{0}} .
$$

This can be verified shifting the profiles in Fig. 3 to overlap them. In Fig. 4 we plot $r$ as a function of $x-u_{f}\left(t-t_{i}\right)$, where $t_{i}$ is any instant after $t_{0}$; it can be seen that the right slopes of the topographies do, in fact, overlap, which means that they move as a traveling wave. In consequence, the visible topography of the plateau can be described by means of a simple formula. For $x>0$ we have

$$
r=\left\{\begin{array}{cc}
r_{0}, & 0 \leq x \leq x_{f} \equiv u_{f}\left(t-t_{0}\right) \\
F\left[x-u_{f}\left(t-t_{0}\right)\right], & x \geq x_{f} .
\end{array}\right.
$$

The topography for $x<0$ is the mirror image of that given by this equation. In Eq. (20) $F=r\left(x, t=t_{0}\right)$ is the relief corresponding to the solution of Eqs. (9)-(15) at the moment of the decoupling. As long as $r$ is small it can be approximated by the self-similar solution (16) and (17) evaluated at $t=t_{0}$. A similar expression for the shape of the sides of the plateau was obtained by Medvedev and Beaumont ${ }^{9}$ based on the channel flow hypothesis. 


\section{DISCUSSION AND CONCLUSIONS}

We have modified our previous model ${ }^{1}$ to reproduce the formation of a plateau due to the decoupling between the basal traction and the crustal flow. To this purpose we assume that at a certain depth $z_{0}$ there is a sharp reduction of the viscosity of the crust. With this change the convergent motion produces a ridge that grows as described in Ref. 1 until the root arrives at $z_{0}$; after that a plateau is formed. The resulting equations are a generalization of those in Ref. 1; however, the velocity profile is linear in the lithospheric mantle as before. On the other hand, the velocity within the crust vanishes under the plateau and has a parabolic profile elsewhere (of course the profile is flat far from the plateau, where the thickness of the crust is approximately $C$ ).

The results of the present model are similar to those derived by means of the channel flow hypothesis. ${ }^{7}$ On the other hand, the calculation based on the last hypothesis assumes a prescribed basal traction acting on the Moho, while in our model the basal traction acts on the level of the lithosphere. This is the main difference between these two approaches and implies that the present model takes into account the dynamics of the entire lithosphere in contrast with the channel flow calculations in which only the crust is considered, while the dynamics of the mantle lithosphere is ignored.

The topography shown in Fig. 3 reproduces the qualitative features of the field data for plateaus such as the Tibet and the Altiplano (see Fig. 1). However, we do not attempt a quantitative comparison because these plateaus are threedimensional objects and our calculations were made assuming planar symmetry.

A perfectly flat plateau whose sides are traveling waves as shown in Figs. 3 and 4 is obtained from the present model if the viscosity drop is extremely abrupt and large, i.e., $n$ $\gg 1$ and $\phi_{0} \ll 1$ (these figures were calculated with $n=1000$ and $\phi_{0}=10^{-5}$ ). If the viscosity change is not so large and/or less abrupt the plateau is not perfectly flat and its escarpments do not behave as an ideal traveling wave (their shape as well as their speed change slightly with time).

Finally, we remark that the present model, besides describing peaked ranges and flat plateaus, can be employed to reproduce other type of orogens by adequate choices of the parameters and can also be generalized to describe nonsymmetrical convergence as well as to include three-dimensional effects. ${ }^{1}$

${ }^{1}$ C. A. Perazzo and J. Gratton, "Convergent flow in a two-layer system and mountain building," Phys. Fluids 22, 056603 (2010).

${ }^{2}$ E. Fielding, B. Isacks, M. Barazangi, and C. Duncan, "How flat is Tibet?,"
Geology 22, 163 (1994).

${ }^{3}$ W. B. Ouimet and K. L. Cook, "Building the Central Andes through axial lower crustal flow," Tectonics 29, C3010 (2010).

${ }^{4}$ K. V. Hodges, in Channel Flow, Ductile Extrusion and Exhumation in Continental Collision Zones, edited by R. D. Law, M. P. Searle, and L. Godin (Geological Society, London, 2006), Vol. 268, pp. 71-90.

${ }^{5}$ L. I. Lobkovsky and V. I. Kerchman, "A two-level concept of plate tectonics: Application to geodynamics," Tectonophysics 199, 343 (1991).

${ }^{6}$ J. P. Avouac and E. B. Burov, "Erosion as a driving mechanism of intracontinental mountain growth," J. Geophys. Res. 101, 17747 (1996).

${ }^{7}$ L. Royden, "Coupling and decoupling of crust and mantle in convergent orogens: Implications for strain partitioning in the crust," J. Geophys. Res., [Solid Earth] 101, 17679 (1996).

${ }^{8}$ M. Gerbault and E. Willingshofer, "Lower crust indentation or horizontal ductile flow during continental collision?," Tectonophysics 387, 169 (2004).

${ }^{9} \mathrm{~S}$. Medvedev and C. Beaumont, in Channel Flow, Ductile Extrusion and Exhumation in Continental Collision Zones, edited by R. D. Law, M. P. Searle, and L. Godin (Geological Society, London, 2006), Vol. 268, pp. 147-164.

${ }^{10} \mathrm{D}$. Li, "Continental lower-crustal flow: Channel flow and laminar flow," Earth Sci. Front. 15, 130 (2008).

${ }^{11}$ L. H. Royden, B. C. Burchfiel, R. W. King, E. Wang, Z. Chen, F. Shen, and Y. Liu, "Surface deformation and lower crustal flow in eastern Tibet," Science 276, 788 (1997).

${ }^{12}$ C. Beaumont, R. A. Jamieson, M. H. Nguyen, and B. Lee, "Himalayan tectonics explained by extrusion of a low-viscosity crustal channel coupled to focused surface denudation," Nature (London) 414, 738 (2001).

${ }^{13}$ F. Shen, L. H. Royden, and B. C. Burchfiel, "Large-scale crustal deformation of the Tibetan plateau," J. Geophys. Res. 106, 6793 (2001).

${ }^{14}$ C. Beaumont, R. A. Jamieson, M. H. Nguyen, and S. Medvedev, "Crustal channel flows: 1 . Numerical models with applications to the tectonics of the Himalayan-Tibetan orogen," J. Geophys. Res., [Solid Earth] 109, 6406 (2004).

${ }^{15}$ M. K. Clark, J. W. M. Bush, and L. H. Royden, "Dynamic topography produced by lower crustal flow against rheological strength heterogeneities bordering the Tibetan plateau," Geophys. J. Int. 162, 575 (2005).

${ }^{16}$ L. Godin, D. Grujic, R. D. Law, and M. P. Searle, in Channel Flow, Ductile Extrusion and Exhumation in Continental Collision Zones, edited by R. D. Law, M. P. Searle, and L. Godin (Geological Society, London, 2006), Vol. 268, pp. 1-23.

${ }^{17}$ L. Husson and T. Sempere, "Thickening the Altiplano crust by gravitydriven crustal channel flow," Geophys. Res. Lett. 30, 1243 (2003).

${ }^{18}$ M. Gerbault, J. Martinod, and G. Hérail, "Possible orogeny-parallel lower crustal flow and thickening in the Central Andes," Tectonophysics 399, 59 (2005).

${ }^{19}$ S. Medvedev, Y. Podladchikov, M. R. Handy, and E. Scheuber, "Controls on the deformation of the Central and Southern Andes (10-35 S): Insight from thin-sheet numerical modeling," in The Andes: Active Subduction Orogeny, edited by O. Oncken, G. Chong, G. Franz, P. Giese, H. Götze, V. A. Ramos, M. R. Strecker, and P. Wigger (Springer, Berlin, 2006), pp. 475-494.

${ }^{20}$ J. D. Buckmaster, "Viscous sheets advancing over dry beds," J. Fluid Mech. 81, 735 (1977).

${ }^{21}$ H. E. Huppert, "The propagation of two-dimensional and axisymmetric viscous gravity currents over a rigid horizontal surface," J. Fluid Mech. 121, 43 (1982).

${ }^{22}$ A. Oron, S. H. Davis, and S. G. Bankoff, "Long-scale evolution of thin liquid films," Rev. Mod. Phys. 69, 931 (1997).

${ }^{23}$ Note that there is a misprint in Eqs. (1) and (2) in Ref. 1. 